\title{
DISTRIBUIÇÃO ESPACIAL DE ATRIBUTOS QUÍMICOS DO SOLO EM ÁREAS SOB DIFERENTES
}

USOS AGRÍCOLAS

Otacilio Silveira Junior' ${ }^{1}$, Antônio Clementino dos Santos ${ }^{2}$, Perlon Maia Santos ${ }^{3}$, José Mario Lopes Rocha ${ }^{4}$, Emerson Alexandrino ${ }^{5}$

\section{RESUMO}

O conhecimento da variação espacial de atributos do solo pode contribuir para identificar os efeitos do uso e manejo aplicados ao solo ao longo do tempo. Neste sentido, objetivou-se estudar as características químicas de Neossolo Quartzarênico Órtico típico sob cinco diferentes tipos de uso. O experimento foi realizado em outubro de 2010, no município de Araguaína - TO, caracterizado como pertencente ao bioma Amazônico. Foi estudada a variabilidade espacial dos seguintes atributos químicos do solo: $\mathrm{pH}, \mathrm{P}, \mathrm{K}^{+}, \mathrm{Ca}^{2+}, \mathrm{Mg}^{2+}, \mathrm{Al}^{3+}$ e matéria orgânica (MO). A área diagnosticada conteve cinco diferentes usos do solo (canavial, pastagem com capoeira em áreas aluviais, pastagem irrigada, pastagem convencional e floresta nativa), sendo todas conexas. As variáveis químicas foram obtidas em malhas regulares de $50 \mathrm{x}$ $50 \mathrm{~m}$, em duas profundidades, $0-10 \mathrm{~cm}$ e $10-20 \mathrm{~cm}$, totalizando 110 amostras de solos. Foi observado que as propriedades químicas são influenciadas pelo uso do solo. A pastagem irrigada e canavial mostraram grande capacidade de extração de nutrientes do solo. Área de capoeira tende a apresentar atributos químicos similares aos da área de floresta nativa. O uso convencional da pastagem promoveu menores alterações nas características químicas do solo. A malha de 50 por 50 $\mathrm{m}$, utilizada para amostragem das características químicas do solo, mosrou-se suficiente para evidenciar a distribuição espacial dos atributos químicos $\mathrm{pH}, \mathrm{K}^{+}, \mathrm{Ca}^{2+}, \mathrm{Mg}^{2+}, \mathrm{Al}^{3+} \mathrm{e} \mathrm{MO}$, mas não é suficiente para apresentar distribuição espacial do $\mathrm{P}$, indicando a necessidade de menor distância entre pontos de amostragem, para que seja detectado.

Palavras-chave: alterações químicas do solo, fertilidade do solo, geoestatística, manejo do solo

\section{ABSTRACT \\ SPATIAL DISTRIBUTION OF SOIL CHEMICAL ATTRIBUTES IN AREAS UNDER DIFFERENT AGRARIAN USES}

Knowledge on spatial variation of soil properties can help to identify the effects of use and management applied to soil over time. In this sense it was sought to study the chemical characteristics of typic quartzipsamment soil submitted to five different types of use. The experiment was conducted in October 2010, in the municipality of Araguaína - TO, characterized as belonging to the Amazon biome. We studied the spatial variability of the following soil chemical properties: $\mathrm{pH}, \mathrm{P}, \mathrm{K}^{+}, \mathrm{Ca}^{2+}, \mathrm{Mg}^{2+}, \mathrm{Al}^{3+}$ and organic matter (MO). The area assessed included five different land uses (sugarcane plantation, pasture with poultry in alluvial areas, irrigated pasture and conventional pasture, native forest), all being related. Chemical variables were obtained in regular grids of $50 \times 50 \mathrm{~m}$, at two depths, $0-10 \mathrm{~cm}$ and $10-20 \mathrm{~cm}$, totaling 110 soil samples. It was observed that the chemical properties are influenced by the soil use. Areas of irrigated pasture and sugarcane showed a great capacity for nutrient extraction by these types of use. Poultry production in alluvial areas tends to show chemical properties similar to native forest, highlighting the natural recovery. The grid of 50 by $50 \mathrm{~m}$ used for sampling of soil chemical characteristics is sufficient to show spatial distribution the chemical properties $\mathrm{pH}, \mathrm{K}^{+}, \mathrm{Ca}^{2+}, \mathrm{Mg}^{2+}, \mathrm{Al}^{3+}$, and $\mathrm{MO}$, but it is not sufficient to present spatial distribution of $\mathrm{P}$, indicating the need for a smaller sampling grids for its detection.

Keywords: changes to soil chemistry, soil fertility, geostatistics, soil management

\section{Recebido para publicação em 13/08/2013. Aprovado em 21/07/2014.}

1 - Zootecnista, Doutorando em Ciência Animal Tropical, UFT/Araguaína-TO, otaciliosilveira@hotmail.com

2 - Engenheiro Agrônomo, Professor Adjunto, UFT/Araguaína-TO, clementino@uft.edu.br

3 - Zootecnista, Doutorando em Ciência Animal Tropical, UFT/Araguaína-TO, perllon_zoo@yahoo.com.br

4 - Zootecnista, Mestre em Ciência Animal Tropical, UFT/Araguaìna-TO,jmrocha18@hotmail.com

5 - Zootecnista, Professor Adjunto, UFT/ Araguaína-TO, e_alexandrino@yahoo.com

442 REVENG

442-455p. ENGENHARIA NA AGRICULTURA, VIÇOSA - MG, V.22 N.5, SETEMBRO / OUTUBRO 2014 


\section{INTRODUÇ̃̃O}

A produtividade é a principal característica almejada nos diversos sistemas de produção agropecuária. Para alcançá-la, uma das técnicas é conhecer e quantificar a variação das propriedades químicas dos solos, uma vez que os mesmos são sistemas dinâmicos e abertos e estão em constantes modificações (SILVA; CHAVES, 2001). O planejamento inadequado na utilização do solo e o conhecimento pouco aprofundado de suas características tem sido constante nos mais diversos sistemas de manejo, principalmente nos modelos de pecuária extensiva (CUNHA et al., 2008).

A variabilidade espacial das características do solo ocorre naturalmente como consequência do processo de formação, podendo ser acentuada pelas ações antrópicas devido aos diferentes usos e manejo aplicados (BROWN et al., 2000). No tocante as características dos nutrientes como fósforo, potássio, cálcio e magnésio apresentam aumentos da variabilidade de suas frações em áreas agropecuárias, em relação às áreas nativas (SANTOS et al., 2010). Este aumento da variabilidade inclui a redução destes elementos em pontos regionalizados, favorecendo a degradação.

A modificação da cobertura vegetal original e a implantação de culturas, aliadas a práticas de manejo errôneas, acabam, muitas das vezes, por romper o equilíbrio do solo, alterando suas propriedades químicas, físicas e biológicas (CENTURION et al., 2001). Como é sabido que o uso agrícola do solo altera sua condição natural, é necessário quantificar e qualificar o tipo de mudança causada por determinado uso, tanto em nível espacial como temporal. Isso pode ser feito através do diagnóstico do ambiente antropizado em relação às áreas não modificadas (floresta nativa) deste mesmo ambiente.

O conhecimento do comportamento das variações químicas no solo possibilita adequar determinada atividade agropecuária à sua aptidão e diagnosticar alterações em relação à condição natural, permitindo apontar manejos que revertam as modificações às condições naturais ou até melhores que esta. Isto pode reduzir significativamente a erosão e a degradação física, química e biológica do solo (BERTOLANI; VIEIRA, 2001).

A variabilidade dos solos nas áreas agropecuárias é pouco considerada, sendo comum a aplicação de práticas homogêneas de manejo para toda uma área heterogênea. Este comportamento ocasiona intervenções aquém ou acima da real necessidade de regiões das áreas cultivadas (CASTRIGNANO et al., 2000). Logo, entender que há variabilidade no solo em função do uso, permite adequar o manejo mais apropriado a cada região dentro do mesmo ambiente. Para isso, é preciso entender como os diferentes tipos de usos podem promover diferentes modificações nas características químicas e físicas do solo. Esta visualização pode ser feita a partir da geoestatística.

A geoestatística é uma ferramenta utilizada na detecção da variabilidade existente no ambiente, permitindo analisar características e seus aspectos aleatórios e espaciais, criando imagem da variabilidade de caracteres, identificando o grau de dependência espacial e fornecendo informações que permitam subsidiar o estudo do fenômeno a ser analisado (SILVA NETO et al., 2011a). Ela pode ser empregada na detecção de locais que estão sofrendo alguma alteração e, a partir daí, determinar as condições de causa e efeito e a restauração do ambiente.

Objetivou-se, com este trabalho, diagnosticar as alterações químicas que ocorrem no solo em função de diferentes tipos de usos e identificar a forma de uso (canavial, pastagem com capoeira, pastagem convencional e pastagem irrigada) que mais preserva ou melhora a condição natural do solo (floresta nativa), bem como aquele que mais altera o solo.

\section{MATERIAL E MÉTODOS}

O estudo foi realizado no período de outubro de 2010, na Universidade Federal do Tocantins, no município de Araguaína, TO, região pertencente ao bioma Amazônico, cujas coordenadas geográficas são $7^{\circ} 06^{\prime} 45^{\prime}$ " S e $48^{\circ} 11^{\prime} 42^{\prime \prime} \mathrm{W}$, com altitude média de $235 \mathrm{~m}$. O clima da região é o AW-Tropical quente e úmido, com verão úmido e período de estiagem no inverno, estando sob domínio climático tropical semiúmido, com estiagem de aproximadamente 4 meses (junho a setembro) e precipitação pluviométrica média anual de $1.800,00 \mathrm{~mm}$.

Para a realização do estudo foi escolhida uma área com mesmo solo, classificado como Neossolo Quartzarênico Órtico típico (EMBRAPA, 2006). Para a classificação, foram abertas trincheiras e realizadas sondagens. As características químicas do solo na condição natural (floresta) estão apresentadas no Quadro 1. É a partir dela que são feitas considerações sob o efeito do manejo e uso do 
Quadro 1. Características químicas e granulométricas de um Neossolo Quartzarênico Órtico sob floresta nativa no bioma Amazônico Tocantinense

\begin{tabular}{|c|c|c|c|c|c|c|c|c|c|c|}
\hline \multirow{3}{*}{ Camada $(\mathrm{cm})$} & \multirow{2}{*}{$\mathrm{pH}$} & \multirow{2}{*}{ MO } & \multirow{2}{*}{$\frac{\text { Disponível }}{\mathrm{P}}$} & \multicolumn{4}{|c|}{ Trocável } & \multirow[t]{2}{*}{ Areia } & \multirow[t]{2}{*}{ Silte } & \multirow[t]{2}{*}{ Argila } \\
\hline & & & & K & $\mathrm{Ca}$ & $\mathrm{Mg}$ & $\mathrm{Al}$ & & & \\
\hline & $\left(\mathrm{CaCl}_{2}\right)$ & $\%$ & $\mathrm{mg} \mathrm{dm} \mathrm{m}^{-3}$ & \multicolumn{4}{|c|}{--------- $\mathrm{cmol}_{\mathrm{c}} \mathrm{dm}^{-3}$-------- } & \multicolumn{3}{|c|}{------------\%\%----------- } \\
\hline $0-10$ & 4,23 & 2,98 & 0,38 & 0,131 & 3,59 & 3,04 & 1,18 & 94,85 & 1,9 & 3,25 \\
\hline $10-20$ & 4,16 & 1,6 & 0,27 & 0,120 & 3,03 & 3,08 & 1,28 & 93,75 & 2,75 & 3,5 \\
\hline
\end{tabular}

solo sob as condições químicas nas áreas vizinhas.

$\mathrm{Na}$ área de estudo foram diagnosticados cinco tipos de uso, ou cobertura do solo: canavial, pastagem com capoeira, pastagem convencional, pastagem irrigada e floresta nativa, que foram divididas em subárea para adequação do estudo (Figura 1). A região sob floresta nativa foi considerada como sendo a de condição natural ou em equilíbrio e, a partir dela, foi inferido se os outros tipos de uso modificaram a condição do solo, tanto para a melhoria quanto para degradação do sistema.

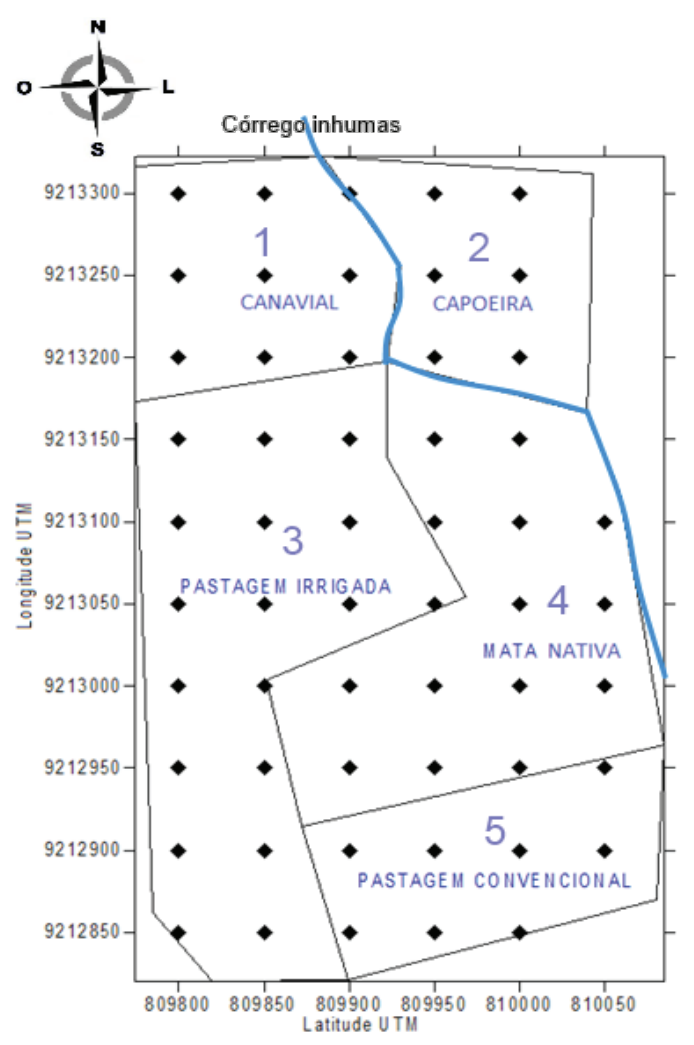

Figura 1. Mapa da área total avaliada com diferentes tipos de uso do solo. (*) Ponto de coleta de informações químicas e física do solo e de biomassa vegetativa.
A subárea sob cultivo de pastagem irrigada apresenta área de 4,23 ha, implantada a mais de 4 anos, sendo destinada à alimentação de gado de leite. Nela é cultivada a gramínea forrageira Brachiaria brizantha, cv. Marandu. Esta subárea, que apresenta declividade média de $4 \%$, foi corrigida na implantação da pastagem com 2,5 t $\mathrm{ha}^{-1}$ de calcário dolomítico. Realizou-se a adubação de manutenção parcelada durante o período das águas ( 8 meses), cujo total anual soma $400 \mathrm{~kg} \mathrm{ha}^{-1}$ de N; $80 \mathrm{~kg} \mathrm{ha}^{-1}$ de $\mathrm{P}_{2} \mathrm{O}_{5}$ e $240 \mathrm{~kg} \mathrm{ha}^{-1}$ de $\mathrm{K}_{2} \mathrm{O}$.

A subárea de Pastagem Convencional, de 2 há, apresenta declividade média de $6 \%$, tendo sido reformada há mais de 5 anos e destinada à alimentação de gado de leite e corte. A cultura implantada é a Brachiaria brizantha, cv. Marandu. Esta subárea nunca foi corrigida e não recebe nenhum tipo de adubação além de não haver controle da taxa de lotação animal.

A subárea de Canavial, de 1,73 há, foi implantada no início de 2010, apresentando declividade média de $3 \%$. Na ocasião do plantio foi realizada a correção do solo com calcário dolomítico (1 ton $\mathrm{ha}^{-1}$ ) e adubação química nitrogenada, potássica e fosfatada, com aplicação do formulado NPK 1015-10 para fornecer $50 \mathrm{~kg}$ de $\mathrm{N} \mathrm{ha}^{-1}, 75 \mathrm{~kg}$ de $\mathrm{P}$ $\mathrm{ha}^{-1}$ e $50 \mathrm{~kg}$ de $\mathrm{K} \mathrm{ha}^{-1}$. Na presente área realizase colheita anualmente e não há adubação de reposição.

A subárea de Pasto com Capoeira encontra-se em processo de restauração natural da vegetação a mais de 5 anos. Ela nunca foi adubada ou corrigida. A pastagem de Brachiaria brizantha cv. Marandu está degradada e praticamente não apresenta participação significativa dentre as espécies presentes no local. Esta área não é destinada para 
uso dos animais, sendo periodicamente inundada por refluxo lateral do Córrego Inhumas e/ou pela precipitação direta ou água subterrânea. Seu alagamento ocorre anualmente durante o período de chuvas, caracterizando sistema de pulso previsível, monomodal e de baixa amplitude de inundação. $\mathrm{O}$ local apresenta pouca ou nenhuma declividade e possui área total de 2,39 ha.

As subáreas de pastagem, canavial e capoeira foram abertas a mais de 15 anos por processos de derrubada e queima. Posteriormente elas foram cultivadas com pastagens sem controle da fertilidade do solo e sem controle do pastejo animal.

A subárea da floresta nativa, de 3,29 há, está situada próximo ao Córrego Inhumas e apresenta declividade média de $3,5 \%$. Parte da área foi utilizada para a exploração de dendê (Elaeis guineensis Jacq.) há mais de 18 anos. Atualmente ela não sofre nenhum tipo de intervenção antrópica. A floresta sobre a área em estudo caracteriza-se como secundária semi-decídua.

As amostras de solo foram coletadas dentro das subáreas de forma a compor uma área total de 13,64 ha, constituída pelos diferentes tipos de uso. Para realização da coleta, foi delineada uma malha regular ou grid de $50 \times 50 \mathrm{~m}$ abrangendo $\mathrm{a}$ área total. Os pontos para coleta no grid foram obtidos através de GPS (Garmin GPSMap 76CSx), considerando as dimensões da área total. Em cada ponto georreferenciado foram coletadas duas amostras simples de solo para formar uma amostra composta nas profundidades de $0-10 \mathrm{~cm}$ e $10-20$ $\mathrm{cm}$, totalizando 110 amostras compostas.

A análise do solo foi realizada no Laboratório de Solos da Universidade Federal do Tocantins EMVZ. As análises químicas foram conduzidas de acordo com o manual de análises químicas de solos, plantas e fertilizantes da EMBRAPA (SILVA, 2009). Foram mensurados os valores de: $\mathrm{pH}\left(\mathrm{em} \mathrm{CaCl}_{2}\right.$ ), Fósforo disponível (P), Potássio trocável $\left(\mathrm{K}^{+}\right)$, extraído em solução Mehlich $1 \mathrm{e}$ quantificados em espectrofotometria e fotometria de chama respectivamente; Cálcio trocável $\left(\mathrm{Ca}^{2+}\right)$, Magnésio trocável $\left(\mathrm{Mg}^{2+}\right)$, Alumínio trocável $\left(\mathrm{Al}^{3+}\right)$ em extração com $\mathrm{KCl} 1$ molar, quantificados por titulação e Matéria Orgânica do solo, quantificada por oxidação com dicromato.
A forma de distribuição das variáveis químicas do solo, em função dos diferentes tipos de uso, foi avaliada por meio de medidas descritivas e análise geoestatística, com construção de semivariogramas e mapas de isolinhas.

As medidas descritivas avaliadas foram: média, mediana, valores máximos e mínimos, amplitude, desvio padrão e coeficiente de assimetria, curtose e coeficiente de variação $(\mathrm{CV})$. Para isso foi utilizado o software Assistat versão 7.5 beta. Empregouse o teste de Kolmogorov-Smirnov (KS) a 5\% de probabilidade para testar a hipótese de normalidade. De acordo com os valores de CV, a variabilidade dessas variáveis foi classificada, segundo Warrick e Nielsen (1980) em baixa $(\mathrm{CV}<12 \%)$, média $(12 \%<\mathrm{CV}<62 \%)$ e alta $(\mathrm{CV}>62 \%)$.

A variabilidade espacial das características do solo da área em estudo foi avaliada pela ferramenta geoestatística. A variabilidade de cada parâmetro do solo foi determinada por meio da construção de seu respectivo semivariograma, com base na pressuposição de estacionariedade da hipótese intrínseca. Os semivariogramas foram ajustados a modelos matemáticos teóricos - esférico, exponencial e gaussiano - para definição dos valores do efeito pepita $\left(\mathrm{C}_{0}\right)$, do alcance $(\mathrm{A})$ e do patamar $\left(\mathrm{C}+\mathrm{C}_{0}\right)$.

O padrão de dependência espacial foi calculado pela semivariância e autocorrelação em função da distância $\gamma($ h) (MATHERON, 1963), utilizando o software $\mathrm{GS}^{+}$(ROBERTSON, 1998), através da Equação 1.

$$
\gamma(h)=\frac{1}{2 N(h)} \sum_{i=1}^{N(h)}\left[Z\left(x_{i}\right) \quad Z\left(x_{i}+h\right)\right]^{2}
$$

em que,

$\gamma(\mathrm{h})=$ semivariância experimental obtida pelos valores amostrados;

$\mathrm{h}=$ distância entre os pontos amostrais;

$\mathrm{N}(\mathrm{h})=$ número de pares de valores medidos $\mathrm{Z}\left(\mathrm{x}_{\mathrm{i}}\right)$, $\mathrm{Z}\left(\mathrm{x}_{\mathrm{i}}+\mathrm{h}\right)$, separados pela distância $\mathrm{h}$; $\mathrm{Z}$ = qualquer um dos parâmetros estudados; $\mathrm{e}$ $\mathrm{X}_{\mathrm{i}}$ e $\mathrm{X}_{\mathrm{i}}+\mathrm{h}=$ posições dos pontos amostrais (VIEIRA et al., 1983).

A seleção do modelo teórico em função das 
variâncias reais foi realizada observando-se a soma do quadrado dos resíduos (SQR), coeficiente de determinação $\left(\mathrm{R}^{2}\right)$, maior grau de dependência espacial (GDE).

Para análise do GDE das variáveis químicas do solo foi utilizada a relação $\mathrm{C} /\left(\mathrm{C}+\mathrm{C}_{0}\right)$, classificada segundo Robertson (1998) como forte (GDE $\geq 0,75)$, moderada $(0,25 \leq \mathrm{GDE}<0,75)$ e baixa dependência (GDE < 0,25), Equação 2.

$$
G D E=\left[\frac{C}{C+C_{0}}\right]
$$

em que,

GDE $=$ grau de dependência espacial;

$\mathrm{C}=$ variância estrutural; e

$\mathrm{C}+\mathrm{C}_{0}=$ patamar

Para verificar a presença de anisotropia, foram calculados semivariogramas para quatro direções, com intervalos de $45^{\circ}\left(0,45,90\right.$ e $\left.135^{\circ}\right)$. Em nenhum dos casos foi evidenciada anisotropia no conjunto dos dados. Logo, foram assumidos semivariogramas com $90^{\circ}$ : isotrópicos.

Após o ajuste dos semivariogramas os dados foram submetidos à construção de mapas através do método de krigagem, considerando a distância e a variância entre pontos vizinhos para a geração de valores de $\mathrm{pH}, \mathrm{P}, \mathrm{K}, \mathrm{Ca}, \mathrm{Mg}, \mathrm{Al}$ e $\mathrm{MO}$ em locais não amostrados.

$\mathrm{O}$ resultado do diagnóstico foi expresso em forma de mapas de isolinhas e a maneira de localizar uma isolinha entre dois pontos foi determinada pela interpolação dos dados, por meio de krigagem ordinária (LANDIM, 2006). O programa Surfer 8.0 (GOLDEN SOFTWARE, 2002) foi utilizado para manipulação e visualização da distribuição espacial, por meio da construção dos mapas de isolinhas das variáveis, de acordo com as coordenadas geográficas. A análise e os mapas foram feitos tratando-se a área de levantada como um todo, sem a divisão em piquetes.

\section{RESULTADOS E DISCUSSÃO}

A análise descritiva dos dados (Quadro 2) revelou a não normalidade das variáveis $\mathrm{pH}, \mathrm{P}, \mathrm{K}, \mathrm{Ca}, \mathrm{Mg}$, $\mathrm{Al}$ e MO, no entanto, medidas de tendência central (média e mediana) não são parâmetros eficientes para caracterizar fenômenos que apresentam dependência espacial por se tratar de estimadores muito influenciados por valores extremos e, assim, capazes de ocultar variações significativas (SILVA et al., 2010). Ao analisarem-se conjuntamente os valores de amplitude, coeficiente de variação e os testes de normalidade para as variáveis em estudo, fica evidente a diferença entre os tipos de uso da área, já que estes promoverem uma variação expressiva em torno da média.

Os coeficientes de assimetria e curtose também demonstram afastamento dos dados em torno da média (valores distantes de zero caracterizam distribuições como não normais) ficando os dados acima dos valores críticos para normalidade. $\mathrm{O}$ teste de Kolmogorov-Smirnov ( $>>0,05)$ evidencia que os dados não apresentaram distribuição normal (Quadro 2) a 5\% de probabilidade, sendo atribuído ao manejo adotado ao solo nos diferentes usos agrário e fatores pedológicos do solo, que influencia facilmente nas características químicas do solo.

De acordo com Paz-Gonzalez et al. (2001), quando é satisfeita a normalidade dos dados, a estimativa de valores em locais não medidos utilizando o método da krigagem na interpolação tem sua eficiência aumentada, apresentando melhores resultados em relação a outros métodos. Contudo, a normalidade da distribuição dos dados não é exigência da geoestatística, apesar de ser interessante que os valores de assimetria não sejam muito distantes de zero, a fim de caracterizar alongamento caudal (FREDDI et al., 2006; CORÁ; BERALDO, 2006). De modo geral, os valores observados são anormais, fator decorrente da excessiva variação da área estudada, relacionado aos efeitos dos diferentes usos do solo e manejos adotados.

A variável $\mathrm{pH}$ apresentou o coeficiente de variação classificado como baixo para as duas profundidades de coleta $0-10$ e 10-20 cm, representando pouca variabilidade para essa variável. Carvalho et al. (2002) encontraram valores muito similares aos observados ( $\mathrm{CV}$ menor que $10 \%$ ). Esse fato se deve a pouca variação na área, além de estar relacionado ao processo de formação do solo e adição de substâncias orgânicas 
Quadro 2. Resumo estatístico descritivo das características químicas nas profundidades 0-10 e 10-20 cm em solos com diferentes usos

\begin{tabular}{|c|c|c|c|c|c|c|c|c|c|c|c|}
\hline \multirow{2}{*}{ Variável } & Mín & Max & $\mathbf{M}$ & Md & Apl & $\mathbf{S}$ & CV(\%) & As & C & K-S & \\
\hline & \multicolumn{11}{|c|}{ Profundidade 0-10 cm } \\
\hline $\mathbf{p H}$ em $\mathrm{CaCl}_{2}$ & 3,67 & 6,42 & 4,47 & 4,35 & 2,75 & 0,53 & 11,85 & 1,51 & 3,38 & $\mathrm{p}<0,02$ & NS \\
\hline MO (\%) & 0,39 & 9,72 & 2,23 & 1,62 & 9,33 & 1,99 & 89,04 & 2,24 & 4,93 & $\mathrm{p}<0,01$ & NS \\
\hline $\mathbf{P}\left(\mathrm{mg} \mathrm{dm}^{-3}\right)$ & 0,23 & 2,98 & 0,63 & 0,47 & 2,75 & 0,47 & 75,13 & 2,67 & 10,03 & $\mathrm{p}<0,01$ & NS \\
\hline $\mathbf{K}^{+}\left(\mathrm{cmol}_{\mathrm{c}} \mathrm{dm}^{-3}\right)$ & 0,02 & 1,45 & 0,18 & 0,12 & 1,43 & 0,24 & 133,38 & 4,26 & 19,69 & $\mathrm{p}<0,01$ & NS \\
\hline $\mathbf{C a}^{2+}\left(\mathrm{cmol}_{\mathrm{c}} \mathrm{dm}^{-3}\right)$ & 0,14 & 13,64 & 2,82 & 1,45 & 13,5 & 3,25 & 115,2 & 1,88 & 2,97 & $\mathrm{p}<0,01$ & NS \\
\hline $\mathbf{M g}^{2+}\left(\mathrm{cmol}_{\mathrm{c}} \mathrm{dm}^{-3}\right)$ & 0,18 & 10,79 & 2,35 & 1,22 & 10,61 & 2,51 & 106,87 & 1,73 & 2,34 & $\mathrm{p}<0,01$ & NS \\
\hline \multirow[t]{2}{*}{$\mathbf{A l}^{3+}\left(\mathrm{cmol}_{\mathrm{c}} \mathrm{dm}^{-3}\right)$} & 0,00 & 5,34 & 0,62 & 0,29 & 5,34 & 0,96 & 154,73 & 3,09 & 11,33 & $\mathrm{p}<0,01$ & NS \\
\hline & \multicolumn{11}{|c|}{ Profundidade $10-20 \mathrm{~cm}$} \\
\hline $\mathbf{p H}$ em $\mathrm{CaCl}_{2}$ & 3,75 & 6,54 & 4,36 & 4,2 & 2,79 & 0,52 & 12,04 & 1,96 & 5,09 & $\mathrm{p}<0,01$ & NS \\
\hline MO (\%) & 0,28 & 3,33 & 1,28 & 1,04 & 3,05 & 0,74 & 57,84 & 1,30 & 1,03 & $\mathrm{p}<0,01$ & NS \\
\hline $\mathbf{P}\left(\mathrm{mg} \mathrm{dm}^{-3}\right)$ & 0,18 & 2,32 & 0,40 & 0,29 & 2,04 & 0,33 & 81,53 & 3,70 & 16,86 & $\mathrm{p}<0,01$ & NS \\
\hline $\mathbf{K}^{+}\left(\mathrm{cmol}_{\mathrm{c}} \mathrm{dm}^{-3}\right)$ & 0,02 & 1,33 & 0,14 & 0,09 & 1,32 & 0,19 & 135.83 & 5,24 & 32,87 & $\mathrm{p}<0,01$ & NS \\
\hline $\mathbf{C a}^{2+}\left(\mathrm{cmol}_{\mathrm{c}} \mathrm{dm}^{-3}\right)$ & 0,16 & 9,69 & 2,05 & 1,45 & 9,53 & 2,32 & 113,12 & 2,00 & 3,19 & $\mathrm{p}<0,01$ & NS \\
\hline $\mathbf{M g}^{2+}\left(\mathrm{cmol}_{\mathrm{c}} \mathrm{dm}^{-3}\right)$ & 0,18 & 8,47 & 1,90 & 0,88 & 8,29 & 2,12 & 111,31 & 1,71 & 2,14 & $\mathrm{p}<0,01$ & NS \\
\hline $\mathbf{A l}^{3+}\left(\mathrm{cmol}_{\mathrm{c}} \mathrm{dm}^{-3}\right)$ & 0,00 & 6,95 & 0,74 & 0,37 & 6,95 & 1,08 & 145,7 & 3,81 & 19,52 & $\mathrm{p}<0,01$ & NS \\
\hline
\end{tabular}

Mín=valor mínimo, $\mathrm{Max}=$ Valor máximo, $\mathrm{M}=$ Média, $\mathrm{Md}=$ Mediana, $\mathrm{Apl}=$ Amplitude, $\mathrm{S}=$ desvio padrão, $\mathrm{CV}=$ coeficiente de variação, As=coeficiente de assimetria, $\mathrm{C}=$ curtose, $\mathrm{K}-\mathrm{S}=$ teste de Kolmogorov-Smirnov, NS=não significativo a $5 \%$ de probabilidade.

em função do uso (Quadro 2). Para o $\mathrm{P}, \mathrm{K}, \mathrm{Ca}$, $\mathrm{Mg}, \mathrm{Al}$ e $\mathrm{MO}$, a variabilidade foi considerada alta para as duas profundidades de coleta, 0-10 e 10$20 \mathrm{~cm}$ (Quadro 2), por sofrerem grande influência do manejo de uso da área devido os efeitos de adição, conservação ou perda do nutriente, que são determinados pelo manejo.

Ao analisar-se o $\mathrm{pH}$ da área como um todo, pode-se observar que os valores médios nas profundidades de $0-10$ e $10-20 \mathrm{~cm}$ apresentam-se de médio a baixo para os diferentes usos. As regiões com maiores valores $(\mathrm{pH}=5,7)$ são referentes a área de canavial, demonstrando o efeito residual do manejo de correção do solo para a implantação da cultura. Os valores mais baixos ( $\mathrm{pH}$ de 3,9 a 4,1) são observados nas áreas de mata nativa, o que confirma a acidez natural desse solo. De acordo com a classificação agronômica da acidez de Alvarez et al. (1999), valores de 5,5 a 6,0 são bons, ou seja, acidez fraca. Já valores inferiores a 4,5 são baixos, representando acidez elevada.

A distribuição do $\mathrm{Al}$ apresentou valores médios de $0,74 \mathrm{cmol}_{\mathrm{c}} \mathrm{dm}^{-3}$ (classificação média), com valores pontuais variando de 0 a $6,95 \mathrm{cmol}_{\mathrm{c}} \mathrm{dm}^{-}$ ${ }^{3}$, evidenciando que partes do terreno apresentam leve toxidade ocasionada por este elemento (Quadro 2). As áreas de floresta nativa e capoeira apresentaram as concentrações mais elevadas, demonstrando a toxicidade natural do solo e a recuperação desta condição após a interferência antrópica. As áreas sob manejo apresentaram uma redução da concentração do $\mathrm{Al}$, principalmente onde foi realizada a correção da acidez. A área de canavial apresentou teor de Al próximo a zero, 
possivelmente devido a correção, ao passo que a área de mata nativa apresentou valores próximos a $2 \mathrm{cmol}_{\mathrm{c}} \mathrm{dm}^{-3}$. As maiores concentrações foram verificadas na profundidade de $10-20 \mathrm{~cm}$, com valores próximos a $3 \mathrm{cmol}_{\mathrm{c}} \mathrm{dm}^{-3}$ na área de floresta nativa. Valores acima de $2 \mathrm{cmol}_{\mathrm{c}} \mathrm{dm}^{-3}$ são considerados muito altos (ALVAREZ et al., 1999).

Os valores de alcance para $\mathrm{Ca}$ e $\mathrm{Mg}$ foram elevados (Quadro 3) com GDE classificado como forte. Isto revela uma variação contínua e dependente entre os diferentes usos, com maiores concentrações nas áreas de floresta e capoeira e menores nas áreas de pastagem, de modo a ocorrer, entre estes ambientes, uma variação gradual. Já $\mathrm{o}$ alcance para o $\mathrm{K}$ foi relativamente alto, mas $\mathrm{o}$ GDE foi classificado como moderado. Isto se dá possivelmente em função de altas concentrações de $\mathrm{K}$ em regiões específicas, com progressiva diminuição até valores próximos a zero, em espaços curtos.

Os valores de alcance para a variável $\mathrm{Mg}$ (Quadro 3) mostraram-se inferiores em relação as demais variáveis $(148,2 \mathrm{~m}$ na camada $0-10$ $\mathrm{cm})$. Isso demonstra uma maior heterogeneidade deste elemento, ou seja, uma maior variação aleatória. Tal fato evidencia que os diferentes usos do solo promovem extrações mais desiguais de $\mathrm{Mg}$ em relação ao $\mathrm{Ca}$ e $\mathrm{K}$ e/ou a capacidade de tamponamento do solo para Ca e K são maiores do que para $\mathrm{Mg}$.

Os maiores valores de alcance foram observados para o $\mathrm{pH}$ em ambas profundidades (aproximadamente $809 \mathrm{~m}$ ). Este fato é comumente documentado, pois o $\mathrm{pH}$ do solo é mais dificilmente alterado devido a capacidade de tamponamento dos solos, conferindo a este atributo maior homogeneidade. Já os mais baixos valores de alcance foram observados para o $\mathrm{Mg}$. A variação revela que a concentração no solo está intimamente relacionada com o tipo de uso do solo, à medida que a distância entre as áreas (canavial, pastagem com capoeira, pastagem convencional, pastagem irrigada e floresta nativa) aumenta, mais acentuado é a concentração de $\mathrm{Mg}$.

O alcance do Al foi bastante parecido nas duas profundidades $(273 \mathrm{~m}$ e $281 \mathrm{~m}$ nas camadas de 0-10 e 10-20 cm respectivamente) (Quadro 3). Isto revela certa homogeneidade (GDE moderada) da distribuição do $\mathrm{Al}$ no solo nas duas camadas, que pode estar relacionado às elevadas concentrações naturais de Al no solo estudado. Tal alcance pode não ter sido maior devido à "quebra" da dependência espacial nas áreas que realiza frequentemente a correção do solo (canavial e pastagem irrigada).

Os alcances para a MO nas camadas de 0 10 e $10-20 \mathrm{~cm}$ de profundidade foi de 290,3 e 200,9 m, respectivamente, classificados como moderado. É de se esperar que a MO, juntamente com o $\mathrm{pH}$ apresente maior uniformidade em sua distribuição no ambiente, logo possui GDE forte e elevado alcance. $\mathrm{O}$ resultado relativamente controverso deste estudo pode estar relacionado à variações abruptas dos teores de $\mathrm{MO}$ sob os diferentes uso, como Canavial e Capoeira. Isto demonstra que há sistemas de uso onde está havendo o acúmulo de $\mathrm{MO}$ e outros onde há perda de $\mathrm{MO}$ (Figura 2F compartimento 1 e 2). $\mathrm{Na}$ região de capoeira, por exemplo, por ser área mais baixo que as outras áreas em estudo, há deposição de sedimentos que influencia nas características químicas do solo. Ainda relacionado ao alcance da dependência espacial, verificou-se que com exceção do $\mathrm{P}$, o número de 55 pontos de coletas pode fornecer informações confiáveis quanto às características químicas do solo. Esses resultados são corroborados com os encontrados por Souza et al. (1997), Carvalho et al. (2002) e Souza et al. (2006), os quais afirmaram que o uso do alcance do semivariograma dos atributos do solo, pode reduzir o número de amostras em relação ao uso dos procedimentos amostrais definidos na estatística clássica, principalmente em grandes áreas, onde a variação dos dados tende a ser maior, devido à maior heterogeneidade do solo.

As variáveis analisadas apresentaram ajustes para os modelos exponenciais e esféricos na sua grande maioria, exceto para $\mathrm{P}$, que apresentou efeito pepita puro nas duas profundidades (Quadro 3). Na profundidade de $10-20 \mathrm{~cm}$, as variáveis melhores se ajustaram aos modelos esféricos. $\mathrm{O}$ modelo esférico ajustado para $\mathrm{pH}, \mathrm{Ca}, \mathrm{Mg}, \mathrm{K}$, na profundidade de $10-20 \mathrm{~cm}$, e Al mais $\mathrm{MO}$ nas duas profundidades analisadas caracteriza a perda de forma mais rápida da semelhança entre as variáveis à medida que se aumenta a distância. Já a falta de ajuste do $\mathrm{Pa}$ algum modelo revela a descontinuidade 
Quadro 3. Modelos e parâmetros estimados aos semivariogramas experimentais para as características químicas do solo nas profundidades de 0-10 e 10-20 cm em diferentes tipos de uso no norte do Tocantins

\begin{tabular}{|c|c|c|c|c|c|c|c|c|}
\hline & Modelo & Co & $\mathrm{Co}+\mathrm{C}$ & Ao & $\begin{array}{c}\mathbf{0} /(\mathrm{Co}+\mathrm{c})] \\
\mathbf{x} 100\end{array}$ & GDE & Classificação & $\mathbf{r}^{2}$ \\
\hline \multicolumn{9}{|c|}{ Profundidade 0-10 cm } \\
\hline $\mathbf{p H}$ em $\mathrm{CaCl}_{2}$ & Exponencial & 0,06 & 1,14 & 809,20 & 4,83 & 0,95 & Forte & 0,99 \\
\hline MO (\%) & Esférico & 1,20 & 4,63 & 290,30 & 25,92 & 0,74 & Moderada & 0,39 \\
\hline $\mathbf{P}\left(\mathrm{mg} \mathrm{dm}^{-3}\right)$ & Pepita puro & 0,24 & 0,24 & 313,81 & 98,05 & 0,00 & Baixa & 0,01 \\
\hline $\mathbf{K}^{+}\left(\mathrm{cmol}_{\mathrm{c}} \mathrm{dm}^{-3}\right)$ & Exponencial & 0,013 & 0,048 & 251,80 & 27,66 & 0,72 & Moderada & 0,66 \\
\hline $\mathrm{Ca}^{2+}\left(\mathrm{cmol}_{\mathrm{c}} \mathrm{dm}^{-3}\right)$ & Exponencial & 2,47 & 21,47 & 367,80 & 11,50 & 0,88 & Forte & 0,98 \\
\hline $\mathbf{M g}^{2+}\left(\mathrm{cmol}_{\mathrm{c}} \mathrm{dm}^{-3}\right)$ & Exponencial & 0,51 & 9,03 & 148,20 & 5,65 & 0,94 & Forte & 0,97 \\
\hline $\mathbf{A l}^{3+}\left(\mathrm{cmol}_{\mathrm{c}} \mathrm{dm}^{-3}\right)$ & Esférico & 0,46 & 1,13 & 273,70 & 40,92 & 0,59 & Moderada & 0,50 \\
\hline \multicolumn{9}{|c|}{ Profundidade $10-20 \mathrm{~cm}$} \\
\hline pH em $\mathrm{CaCl}_{2}$ & Esférico & 0,07 & 0,659 & 810,8 & 10,77 & 0,892 & Forte & 0,99 \\
\hline MO $(\%)$ & Esférico & 0,11 & 0,616 & 200,9 & 18,51 & 0,715 & Moderado & 0,96 \\
\hline $\mathbf{P}\left(\mathrm{mg} \mathrm{dm}^{-3}\right)$ & Pepita puro & 0,12 & 0,115 & 313,81 & 100 & 0,00 & Baixa & 0,06 \\
\hline $\mathbf{K}^{+}\left(\mathrm{cmol}_{\mathrm{c}} \mathrm{dm}^{-3}\right)$ & Esférico & 0,009 & 0,027 & 175,5 & 33,08 & 0,669 & Moderada & 0,78 \\
\hline $\mathrm{Ca}^{2+}\left(\mathrm{cmol}_{\mathrm{c}} \mathrm{dm}^{-3}\right)$ & Esférico & 1,29 & 5,840 & 185,4 & 22,09 & 0,77 & Forte & 0,99 \\
\hline $\mathbf{M g}^{2+}\left(\mathrm{cmol}_{\mathrm{c}} \mathrm{dm}^{-3}\right)$ & Esférico & 0,57 & 5,163 & 195,2 & 11,04 & 0,89 & Forte & 0,95 \\
\hline $\mathbf{A l}^{3+}\left(\mathrm{cmol}_{\mathrm{c}} \mathrm{dm}^{-3}\right)$ & Esférico & 0,47 & 1,457 & 281,6 & 32,53 & 0,675 & Moderada & 0,44 \\
\hline
\end{tabular}

$\mathrm{Ca}^{2+}=$ Cálcio, $\mathrm{Mg}^{2+}=$ Magnésio, $\mathrm{K}^{+}=$Potássio, $\mathrm{P}=$ Fósforo, $\mathrm{Al}^{3+}=$ Alumínio, $\mathrm{Co}=$ =feito pepita, $\mathrm{Co}+\mathrm{C}=$ Patamar, $\mathrm{Ao}=$ Alcance, $[\mathrm{Co} /(\mathrm{Co}+\mathrm{c})] \times 100=$ Contribuição do efeito pepita $(\%), \mathrm{GDE}=$ Grau de Dependência Espacial, $\mathrm{r}^{2}=$ coeficiente de determinação.

e a distribuição totalmente aleatória do elemento, tanto dentro de toda área estudada como dentro de cada subárea de uso do solo. Este fato pode ser o resultado de diferentes exigências de cada cultura e da exportação da cultura, de adubações em áreas muito específicas e de perdas e/ou complexação em pontos distintos.

Devido à falta de ajuste do semivariograma para $P$ em função da aleatoriedade da distribuição deste nutriente, a krigagem não foi executada, não sendo gerado o mapa de isolinhas para este elemento.

A falta de ajuste para o atributo $P$ indica grande capacidade de dispersão dos dados nas áreas estudadas, que pode ser devido a variações não detectadas com a distância entre as amostragens (malha $50 \times 50 \mathrm{~m}$ ), sendo insuficiente para estudar a variabilidade espacial do $\mathrm{P}$ nessa situação, uma vez que em Neossolo Quartzarênico possui baixa disponibilidades de agentes ligantes no solo, facilitando as perdas, tornando nesse tipo de solos dependentes de suprimentos de P. De acordo com o relato de Vieira (2000), na existência de menor distância entre os pontos, haverá também maior facilidade para as variáveis apresentarem dependência espacial.

Um parâmetro importante a ser observado 


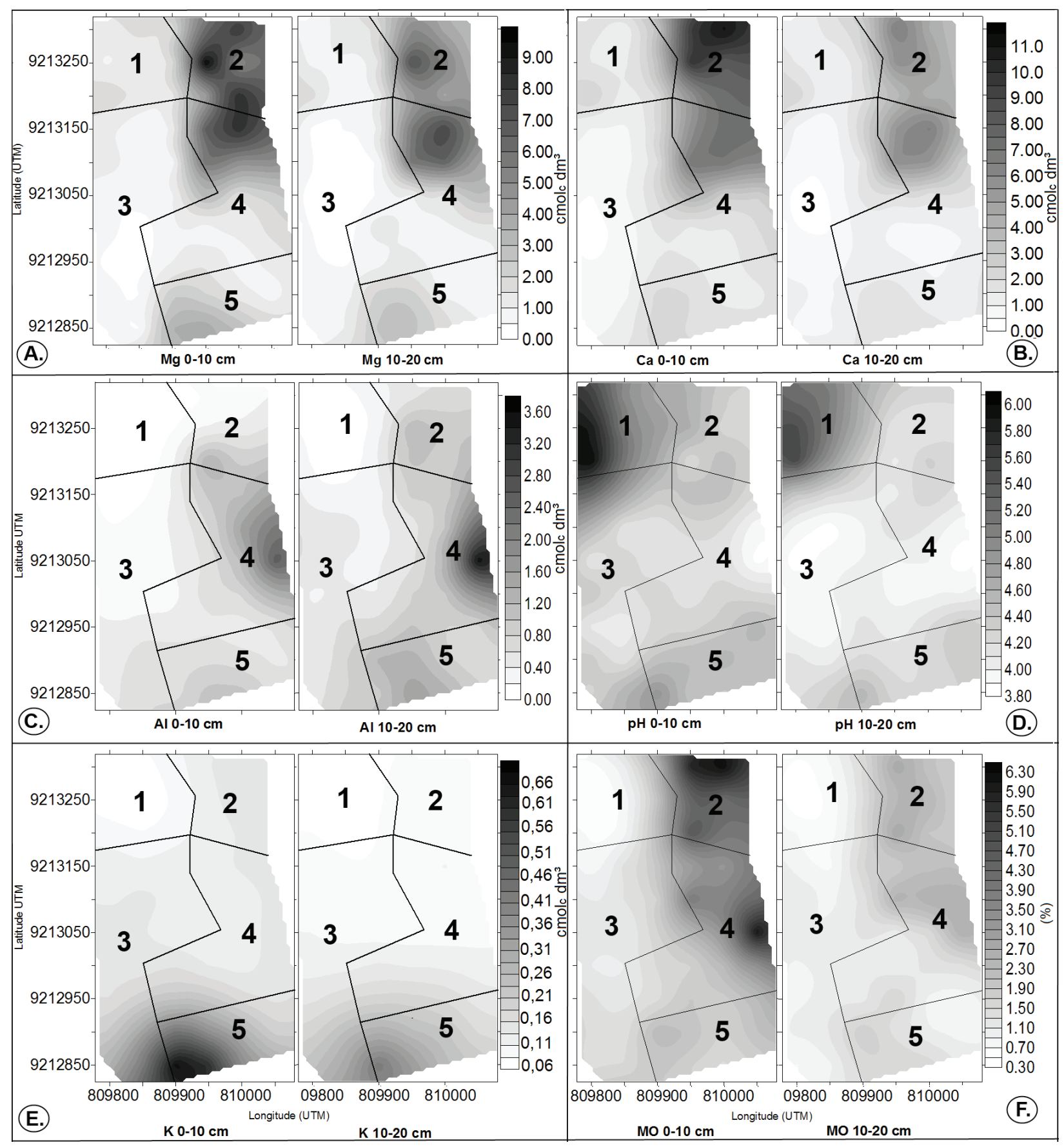

Figura 2. Distribuição espacial do $\mathrm{Mg}^{2+}\left(\mathrm{cmol}_{c} \mathrm{dm}^{-3}\right)$ compartimento " $\mathrm{A}$ "; $\mathrm{Ca}^{2+}\left(\mathrm{cmol}_{\mathrm{c}} \mathrm{dm}^{-3}\right)$ compartimento "B"; $\mathrm{Al}^{3+}\left(\mathrm{cmol}_{\mathrm{c}} \mathrm{dm}^{-3}\right)$ compartimento "C"; $\mathrm{pH}$ compartimento " $\mathrm{D}$ "; $\mathrm{K}^{+}\left(\mathrm{cmol}_{\mathrm{c}} \mathrm{dm}^{-3}\right)$ compartimento "E"; M.O. (\%) compartimento "F", nas profundidades de 0-10 e 10-20 cm. Áreas 1, 2, 3, 4, 5, representam diferentes usos do solo, a saber: 1: Canavial; 2: Capoeira em solos aluviais; 3 : Pastagem irrigada; 4: Floresta nativa; 5: Pastagem convencional

na análise dos componentes do semivariograma (Quadro 3.), é o efeito pepita $\left(\mathrm{C}_{0}\right)$, que representa a variância não explicada ou ao acaso, frequentemente causada por erros de medições ou variações dos atributos que não podem ser detectadas na escala de amostragem (VIEIRA, 2000). Para o P, nas profundidades de $0-10$ e $10-20 \mathrm{~cm}$, o erro ou efeito pepita chega a ser quase a totalidade da variação.

$\mathrm{O}$ efeito pepita para as variáveis $\mathrm{pH}, \mathrm{Ca} \mathrm{e}$

\section{REVENG \\ 442-455p.}

ENGENHARIA NA AgRiCUlturA, VIÇOSA - MG, V.22 N.5, SETEMBRO / OUTUBRO 2014 
Mg não ultrapassaram 22,9\%, mostrando que a maior variação destes atributos é explicada pela dependência espacial observada nos valores de GDE acima de 0,75 . Isto implica que as variáveis têm uma distribuição não casual dentro da área em estudo e variam de acordo com o uso do solo. Já os elementos $\mathrm{K}, \mathrm{Al}, \mathrm{P}$ apresentaram maiores valores de efeito pepita, indicando menor dependência espacial (Quadro 3.), consequentemente, estes elementos variam de forma mais independente ao tipo de uso do solo.

A observância dos mapas de isolinhas (Figura $2 \mathrm{~A}$ e 2B), revela que os maiores teores de $\mathrm{Mg}$ e $\mathrm{Ca}$ encontram-se na camada superior do solo, na profundidade de $0-10 \mathrm{~cm}$, havendo pouca redução com o aprofundamento para a camada de $0-20 \mathrm{~cm}$, e que não há comportamento homogêneo na sua distribuição, sugerindo a existência de diferentes zonas de manejo na área: efeito do uso do solo. Além disso, foram observados os maiores teores deste nutriente nas áreas onde o solo possui umidade superior, áreas 2 e 4 (Capoeira em solos aluviais e Mata nativa, respectivamente). Isto ocorre principalmente em função destas áreas possuírem características de deposição de sedimentos, havendo assim arraste desse elemento pela água das chuvas através do escoamento superficial da parte alta para a mais baixa.

Os teores de $\mathrm{Ca}$ e $\mathrm{Mg}$ apresentaram uma distribuição similar na área total em ambas as profundidades. As maiores concentrações foram verificadas nas áreas de Capoeira e Floresta, com variações de 4 a $11 \mathrm{cmol}_{c} \mathrm{dm}^{-3}$ para o Ca e de 4 a $9 \mathrm{cmol}_{\mathrm{c}} \mathrm{dm}^{-3}$ para o $\mathrm{Mg}$. De acordo com Alvarez et al. (1999), valores acima de $4 \mathrm{cmol}_{\mathrm{c}} \mathrm{dm}^{-3}$ para $\mathrm{Ca}$ e $1,5 \mathrm{cmol}_{\mathrm{c}} \mathrm{dm}^{-3}$ para $\mathrm{Mg}$ são muito bons. As áreas de pastagem apresentaram teores de $\mathrm{Ca}$ de médio a baixo ( 0 a $2 \mathrm{cmol}_{\mathrm{c}} \mathrm{dm}^{-3}$ para o Ca). As menores concentrações foram detectadas na área de pastagem irrigada (0 a $1,5 \mathrm{cmol}_{\mathrm{c}} \mathrm{dm}^{-3}$ para $\mathrm{Ca}$ ), fato que pode ser explicado devido a maior intensidade de exportação dos nutrientes em função da intensificação da exploração. A área de canavial apresentou valores de médio a bom $\left(1,5\right.$ a $3 \mathrm{cmol}_{\mathrm{c}}$ $\mathrm{dm}^{-3}$ ) possivelmente devido a correção do solo.

Os teores de $\mathrm{Mg}$ nas áreas de pastagem foram considerados de médio a bom $\left(0,5\right.$ a $1,5 \mathrm{cmol}_{\mathrm{c}}$ $\mathrm{dm}^{-3}$ ), com exceção da área de pastagem irrigada que apresentou valores baixos (de 0 a $0,5 \mathrm{cmol}_{\mathrm{c}}$ $\mathrm{dm}^{-3}$ ), fato também atribuído a maior exportação de nutrientes nesta área devido a intensificação. A área de canavial apresentou valores de bom a muito bom ( $1 \mathrm{a} 2 \mathrm{cmol}_{\mathrm{c}} \mathrm{dm}^{-3}$ ) devido a correção do solo com calcário dolomítico.

Nos mapas do elemento Al, foi observada certa similaridade nas duas profundidades, sendo que os maiores teores se mostraram principalmente no local onde não existiu nenhuma interferência antrópica quanto ao uso, ou seja, na mata nativa (Figura 2. Compartimento 4). Pereira et al. (2000) relataram que a solubilidade do alumínio aumenta com a redução do $\mathrm{pH}$. Isto é bem nítido nas duas profundidades, onde nos locais com menores valores de $\mathrm{pH}$ houveram maiores concentrações de $\mathrm{Al}$ (Figura 2C e 2D) (PRADO; NATALE, 2004).

$\mathrm{Na}$ área de floresta nativa foi observado os menores valores de $\mathrm{pH}$ nas duas profundidades (Figura 2D). Isto ocorre, em parte, devido a maior aporte de serrapilheira, que em seu processo de decomposição libera quantidades significativas de ácidos orgânicos (Húmicos e Flúvicos) contribuindo assim para a diminuição do $\mathrm{pH}$ do solo (MORAES et al., 2008). A elevação do pH a valores superiores a 5,5 faz com que as formas trocáveis de $\mathrm{Al}$ transformem-se em $\mathrm{Al}(\mathrm{OH})_{3}$, tornando-o na forma neutra e insolúvel, reduzindo drasticamente sua atividade (ANDRADE, 2011).

Em relação à concentração dos elementos avaliados nas duas profundidades, pode-se notar que os valores de $\mathrm{Ca}, \mathrm{K}, \mathrm{pH}$ e $\mathrm{MO}$ são mais elevados na camada de $0-10 \mathrm{~cm}$ de profundidade. Esta relação pode ser explicada pelos efeitos de correção do solo nas áreas de canavial e pastagem irrigada e maior deposição de resíduos orgânicos na superfície do solo nas áreas de floresta nativa e capoeira.

$\mathrm{Na}$ área de canavial foram verificados os menores valores de $\mathrm{Al}$ e, inversamente, os maiores valores de $\mathrm{pH}$. Isto ocorreu devido a área ter recebido correção do solo com a aplicação de calcário no momento da implantação da cultura, contribuindo esta técnica para o aumento significativo do $\mathrm{pH}$ (Figura 2D e Figura 2C), além de tornar o $\mathrm{Al}$ insolúvel e na forma neutra nas camadas superficiais do solo (FRAZÃO et al., 2008).

Os teores de $\mathrm{K}$ no solo apresentaram, em ambas 
as profundidades, maior concentração nas áreas de pastagem irrigada e pastagem convencional, sendo, principalmente, os maiores teores observados na camada de $0-10 \mathrm{~cm}$ (Figura 2E). Nestas áreas, os teores foram classificados como sendo de médio a muito bom $\left(0,16 \mathrm{a}>0,31 \mathrm{cmol}_{\mathrm{c}} \mathrm{dm}^{-}\right.$ $\left.{ }^{3}\right)$. Os valores estiveram muito concentrados em uma única região comum as duas áreas (extremo sul da pastagem irrigada e sudoeste da pastagem convencional) (Figura 2). Esta concentração se deve ao fato de neste local haver dois pontos atrativos aos animais: o bebedouro e a sombra. Estas estruturas promovem aglomeração dos animais, com consequente aumento na deposição de fezes e urinas neste local (SILVA NETO et al., 2011b), de modo a elevar os teores de K no solo desta região, fato esse observado por Silva et al. (2013).

Os menores valores de $\mathrm{K}$ foram observados na área de canavial, com concentrações de 0,06 a 0,11 $\mathrm{cmol}_{\mathrm{c}} \mathrm{dm}^{-3}$, sendo considerado baixo (ALVAREZ et al., 1999). Este fato pode estar associado a grande exportação do elemento no processo de colheita da cana e evidenciar que a adubação potássica de reposição está sendo insuficiente. Nas áreas de Capoeira e Floresta os teores de $\mathrm{K}$ foram considerados médios na camada de $0-10 \mathrm{~cm}$ de profundidade $\left(16\right.$ a $\left.21 \mathrm{cmol}_{\mathrm{c}} \mathrm{dm}^{-3}\right)$ e de baixo a médio $\left(0,06\right.$ a $\left.0,13 \mathrm{cmol}_{\mathrm{c}} \mathrm{dm}^{-3}\right)$ na camada de $10-$ $20 \mathrm{~cm}$ de profundidade.

As menores concentrações de $\mathrm{K}$ foram observadas na área de canavial, embora esta área tenha recebido adubação para manutenção da cultura. Provavelmente, a correção do solo incorreu a maior disponibilização dos nutrientes, favorecendo maior absorção por parte da cultura (cana-de-açúcar), além da umidade no perfil do solo ter facilitado a movimentação deste elemento por fluxo de massa. Além disso, o corte da cana e a remoção do material da área promoveu elevada exportação deste nutriente. Kaminski et al. (2005), trabalhando com correção do solo, verificou que a adição de calcário elevou a saturação por bases do solo, ocasionando a disponibilização de alguns nutrientes.

A porcentagem de matéria orgânica do solo (MO) foi mais elevada nas áreas de Capoeira e Floresta, e os maiores teores ocorreram na camada de $0-10 \mathrm{~cm}$ de profundidade (Figura $2 \mathrm{~F}$ ), sendo considerados de médio a muito bom $(2,5$ a $6 \%)$ (ALVAREZ et al., 1999). O acúmulo de matéria orgânica na área de Capoeira pode ser explicado pelo fato de que neste local existe constante deposição de sedimentos carreados através da água das chuvas, havendo, assim, forte tendência desse material em se manter nas camadas mais externas do solo. Já na área de Floresta nativa o acúmulo de material orgânico é função do grande e intenso fluxo de serrapilheira advindo da vegetação, proporcionando maior cobertura do solo, principalmente nas camadas mais superficiais, além de favorecer maior ciclagem de nutrientes neste ambiente (SCHROTH, 2002).

As áreas de pastagens apresentaram teores de MO classificados como baixos $(<2 \%)$ em ambas as profundidades. Este fato revela problemas relacionados à taxa de lotação, pressão de pastejo e eficiência de pastejo, pois, apesar de ser a pastagem considerada como uma acumuladora de resíduos orgânicos devido a deposição de seus tecidos foliares e radiculares no solo, o superpastejo pode acelerar a degradação dos resíduos orgânicos, impedir que parte dos tecidos da planta retornem ao solo e reduzir o crescimento de raízes devido à exaustão das reservas das plantas.

A área de Canavial exibiu os menores percentuais de $\mathrm{MO}$ em ambas as profundidades, sendo classificada como muito baixa $(<0,7 \%)$ (ALVAREZ et al., 1999). Este fato revela que este sistema de uso do solo pode reduzir sua qualidade em função da remoção completa da planta (folhas e colmo) para a confecção de silagem. Neste sistema, como a cana de açúcar é utilizada para alimentação animal, não há despalha com uso de fogo, havendo retorno da palhada senescente ao solo. No entanto, a cobertura do solo é baixa, o que favorece as perdas de nutrientes transportados para áreas mais baixas (Figura 2, área 1 e 2).

Atenção especial deve ser dada as áreas de intensificação do uso. A área sob pastagem irrigada (área 3 da Figura 2), consequentemente sob maior intensificação de uso, apresentou valores baixos para concentração de $\mathrm{Ca}, \mathrm{Mg}$ e MO e intermediários para a concentração de $\mathrm{K}$. Isto demonstra o elevado efeito da remoção dos nutrientes via pastejo animal, haja vista que tal 
área recebe adubação de reposição periodicamente, contudo os valores destas bases não são elevados. Este fato ainda demonstra que a intensificação da produção a pasto requer monitoramento regular e reposição periódica de bases. Os baixos teores de $\mathrm{Ca}$ e $\mathrm{Mg}$ são o resultado de uma única calagem realizada na implantação da pastagem, já que nesta mesma área há baixos valores de $\mathrm{pH}$.

\section{CONCLUSÕES}

- As propriedades químicas são influenciadas pelo tipo de uso do solo. As áreas de pastagem irrigada e canavial se mostraram mais diferenciadas das demais, devido à intensificação da ação antrópica, mostrando a grande capacidade de extração de nutriente por estes tipos de uso;

- O uso menos intensivo da pastagem convencional promove menores alterações nas características químicas do solo, fazendo com que se pareça com o solo nativo sob cobertura vegetal natural (floresta);

- O desuso de área com consequente permissão do crescimento de Capoeira promove a reconstituição química do solo, ao longo do tempo.

- A utilização da malha de 50 por 50 m é suficiente para mostrar a distribuição espacial dos atributos químicos $\mathrm{pH}$, teores trocáveis de $\mathrm{K}, \mathrm{Ca}, \mathrm{Mg}, \mathrm{Al}$ e de MO, mas não é para o P disponível, devido sua baixa variabilidade espacial.

\section{AGRADECIMENTOS}

Ao CNPq e a CAPES pelo apoio com a) Bolsas no País / Mestrado; Iniciação Científica - Edital MCT/CNPq n. ${ }^{\circ}$ 12/2010 - IC;b) Edital MCT/CNPq 10/2010 - Apoio Técnico / Edital MCT/CNPq 10/2010 - AT- NS (Nível Superior); e c) Bolsas no País / Produtividade em Pesquisa - PQ - 2009 Orientador (COAGR/CGAPB/DABS). Redes Nacionais de Pesquisa em Agrobiodiversidade e Sustentabilidade Agropecuária - REPENSA/ CNPq; Programa de Apoio a Núcleos de Excelência - PRONEX/SECT/CNPq. Apoio fundamental para realização deste trabalho.

\section{REFERÊNCIAS BIBLIOGRÁFICAS}

ALVAREZ, V.H.; NOVAIS, R.F.; BARROS, N.F. CANTARUTTI, R.B. LOPES, A.S. Interpretação dos resultados de análise de solos. In: RIBEIRO, A.C.; GUIMARÃES, P.T.G.; ALVAREZ, V.H. Recomendações para o uso de corretivos e fertilizantes em Minas Gerais $-5^{\circ}$ Aproximação. Comissão de Fertilidade do Solo do Estado de Minas Gerais - CFSEMG. Viçosa, 1999, p.25-32.

Andrade, M.W. Fontes e doses de N, P, Ke métodos de enxertia na produção de mudas de umbuzeiro (Spondias tuberosa Arr. Cam.). 2011. 142f. Tese (Doutorado em Fitotecnia). Universidade Federal Rural do Semiárido, Mossoró, 2011.

BERTOLANI, F.C; VIEIRA, S.R. Variabilidade espacial da taxa de infiltração de água e da espessura do horizonte A, em um Argissolo Vermelho-amarelo, sob diferentes usos. Revista Brasileira de Ciência do Solo, Viçosa, v.25, n.4, p.987-995, 2001.

BROWN, S; SCHREIER, H; SHAH, P.B. Soil phosphorus fertility degradation: a Geographic Information System-based assessment. Journal of Environmental Quality, Madison, v.29, n.4, p.1152-1160, 2000.

CARVALHO, J.R.P; SILVEIRA, P.M.; VIEIRA, S.R. Geoestatísticana determinação da variabilidade espacial de características químicas do solo sob diferentes preparos. Pesquisa Agropecuária Brasileira, Brasilia, v.37, n.8, p.1151-1159, 2002.

CASTRIGNANÒ, A; GIUGLIARINI, L; RISALITI, R; MARTINELLI, N. Study of spatial relationships among some soil physicochemical properties of a field in central Italy using multivariate geostatistics. Geoderma, Amsterdam, v.97, n.1-2, p.39-60, 2000.

CENTURION, F.J; CARDOSO, P.J; NATALIE, W. Efeito de formas de manejo em algumas propriedades físicas e químicas em Latossolo Vermelhor em diferentes Agroecossistemas. Revista Brasileira de Engenharia Agrícola e 
Ambiental, Campina Grande, v.5, n.2, p.254-258, 2001 .

CORÁ, J.E.; BERALDO, J.M.G. Variabilidade espacial de atributos do solo antes e após calagem e fosfatagem em doses variadas na cultura de canade-açúcar. Engenharia Agrícola, Jaboticabal, v.26, n.2, p.374-387, 2006.

CRESSIE, N. Geoestatistics. The American Statistician, Albuquerque, v.43, n.4, p.197-202, 1989.

CUNHA, N.R.S; LIMA, J.E; GOMES, M.F.M; BRAGA, M.J. A intensidade da exploração agropecuária como indicador da degradação ambiental na região dos Cerrados, Brasil. Revista de Economia e Sociologia Rural, Brasília, v.46, n.2, p.291-323, 2008.

EMBRAPA. Centro Nacional de Pesquisa de Solos. Sistema Brasileiro de Classificação de Solos. $2^{\circ}$ ed. Rio de Janeiro: Embrapa Solos. 2006. 305 p.

FRAZÃO, L.A; PICCOLO, M.C; FEIGL, B.J; CERRI, C.C; CERRI, C.E.P. Propriedades químicas de um Neossolo Quartzerênico sob diferentes sistemas de manejo no Cerrado MatoGrossense. Pesquisa Agropecuária Brasileira, Brasília, v.43, n.5, p. 641-648, 2008.

FREDDI, O.S; CARVALHO, M.P; VERONESI JÚNIOR, V; CARVALHO, G.J. Produtividade do milho relacionada com a resistência mecânica à penetração do solo sob preparo convencional. Engenharia Agrícola, Jaboticabal, v.26, n.1, p.113-121, 2006.

GOLDEN SOFTWARE. Surfer for Windows version 8.0. Colorado: Golden, 2002.

LANDIM, P.M.B. Sobre Geoestatística e mapas. Terra e Didática, Campinas, v.2, n.1, p.19-33, 2006.

KAMINSKI， J; SANTOS, D.R; GATIBONI, L.C; BRUNETTO, G; SILVA, L.S. Eficiência da calagem superficial e incorporada precedendo o sistema plantio direto em um argissolo sob pastagem natural. Revista Brasileira de Ciência do Solo, Viçosa, v.29, n.4, p.573-580, 2005.

KRIGE, D.G.A. statistical approach to some basic mine evalution problems on the witwatersrand. Journal of the Chemical, Metallurgical and Mining Society of South Africa, Marshalltown, v.52, n.6, p.119-139, 1951.

MATHERON, G. Principles of Geostatictics. Economic Geology, Littleton, v.58, n.8, p. 12461266. 1963.

MORAES, L.F.D; CAMPELLOS, E.F.C; PEREIRA, M.G; LOSS, A. Características do solo na restauração de áreas degradadas na reserva biológica de poço das antas, RJ. Ciência Florestal, Santa Maria, v.18, n.2, p.193-206, 2008.

PAZ-GONZALEZ, A; TABOADA CASTRO, M.T.; VIEIRA, S.R. Geostatistical analysis of heavy metals in a one-hectare plotunder natural vegetation in a serpentine area. Canadian Journal of Soil Science, Ottawa, v.81, n.4, p.469-479, 2001.

PRADO, R.M; NATALE, W. Calagem na nutrição de cálcio e no desenvolvimento do sistema radicular da goiabeira. Pesquisa Agropecuária Brasileira, Brasília, v.39, n.10, p.1007-1012, 2004.

PEREIRA, W.L.M; VELOSO, C.A.C; GAMA, J.R.N.F. Propriedades químicas de um latossolo amarelo cultivado com pastagens na Amazônia Oriental. Scientia Agricola, Piracicaba, v.57, n.3, p.531-537, 2000.

ROBERTSON, G.P. GS+: Geostatistics for the environmental sciences - GS+ User's guide. Plainwell, Gamma Design Soffware, 1998. 152p.

SANTOS, A.C; SALCEDO, I.H.; CANDEIAS, A.L.B. Variabilidade espacial da fertilidade do solo sob vegetação nativa e uso agropecuário: estudo de caso na microbacia vaca - PB. Revista Brasileira de Cartografia, Rio de Janeiro, v.2, n. 62, p. 119$124,2010$. 
SCHROTH, G. Conversion os secondary Forest into agroforestry and monoculture plantations in Amazônia: consequences for biomass, litter and soil carbon stocks after 7 years. Forestry Ecology and Managenment, v.163, n.1-3, p.131-150, 2002.

SILVA, F.C. Manual de análises químicas de solos, plantas e fertilizantes. Brasília, DF. Embrapa. $2^{\text {a }}$ Edição., 2009. 624p.

SILVA, F.D; AMADO, T.J.C; BREDEMEIER, C; BREMM, C; ANGHINONI, I; CARVALHO, P.C.F. Pasture grazing intensity and presence or absence of cattle dung input and its relationships to soybean nutrition and yield in integrated croplivestock systems under no-till. European Journal of Agronomy, Amsterdam, v.57, p.84-91, 2013.

SILVA, P.C.M; CHAVES, L.H.G. Avaliação e variabilidade espacial de fósforo, potássio e matéria orgânica em Alissolos. Revista Brasileira de Engenharia Agrícola e Ambiental, Campina Grande, v.3, n.5, p.431-436, 2001.

SILVA, S.A; LIMA, J.S.S; ALVES, A.I. Estudo espacial do rendimento de grãos e porcentagem de casca de duas variedades de Coffea arábica $L$. visando a produção de café de qualidade. Bioscience Journal, Uberlândia, v.26, n.4, p.558$565,2010$.

SILVA NETO, S.P; SANTOS A.C; LIMA LEITE, R.L; DIM, V.P; CRUZ, R.S; PEDRICO, A; NEVES NETO, D.N. Análise espacial de parâmetros da fertilidade do solo em região de ecótono sob diferentes usos e manejos. Semina: Ciências Agrárias, Londrina, v.32, n.2, p. 541-552, 2011a.

SILVA NETO, S.P.; SILVA, R.G.; SANTOS, A.C.; GAMA, F.R.; GUERRA, M.S.S.; BRITO, M.J.D. Padrões espaciais de deposição de fezes por bovinos de corte em áreas de pastagem. Revista Brasileira de Saúde Produção Animal, Salvador, v.12, n.2, p.538-550, 2011 b.

SOUZA, L.S; COGO, N.P; VIEIRA, S.R. Variabilidade espacial de propriedades físicas e químicas do solo em um pomar cítrico. Revista Brasileira de Ciência do Solo, Viçosa, v.21, n.3, p.367-372, 1997.

SOUZA, Z.M.; MARQUES JÚNIOR, J.; PEREIRA, G.T.; MONTANARI, R. Otimização amostral de atributos de latossolos considerando aspectos solo-relevo. Ciência Rural, Santa Maria, v.36, n.3, p.892-836, 2006.

VIEIRA, S.R. Geoestatística em estudos de variabilidade espacial do solo. In. NOVAES, R. F.; ALVAREZ V.V.H.; SCHAEFER, C.E.G.R. Tópicos em ciências do solo. Viçosa, MG: Sociedade Brasileira de Ciência do Solo, v.1, p.1$54,2000$.

WARRICK, A.W.; NIELSEN, D.R. Spatial variability of soil physical properties in the field. In: HILLEL, D. (Ed.). Applications of soil physics. New York: Academic, 1980, p.319-344. 